\title{
Selbst- und Fremdbild der Patientinnen und Patienten im Spital
}

\author{
W. Widmer
}

Patientinnen und Patienten im Spital haben ein Selbstbild, eine Vorstellung von ihrer Rolle. Dieses Selbstbild prägt ihr Verhalten gegenüber den Ärztinnen, Ärzten und Pflegenden. Auch die Spitalmitarbeitenden haben in ihrem Kopf Bilder der Patientinnen und Patienten. Dieses Fremdbild prägt ihr Verhalten gegenüber den Kranken.

Das Selbstbild der Patientin definiert gleichzeitig auch ihre Erwartung an die Rolle des Arztes, und umgekehrt prägt das Bild vom Patienten in den Köpfen der Spitalmitarbeitenden ihre Rollenerwartung an ihn.

Wenn sich Selbst- und Fremdbild nicht decken, kann sich die professionelle Arbeit nicht optimal entfalten. Darum ist es wichtig, dass Ärztinnen, Ärzte und Pflegende ein Sensorium entwickeln für die Selbstbilder der Patienten und ihr Verhalten ihnen gegenüber entsprechend gestalten. Letztlich geht es darum, den Patienten zu befähigen, möglichst autonom mit seiner Krankheit umzugehen und als wesentlicher Partner in seinem Gesundungsprozess mitzuwirken.

Korrespondenz:

Dr. rer. pol. Werner Widmer Stiftung Diakoniewerk Neumünster Schweizerische Pflegerinnenschule Trichtenhauserstrasse 24 CH-8125 Zollikerberg

E-Mail:

werner.widmer@

diakoniewerk-neumuenster.ch
Die Selbstbilder der Patientinnen haben sich im Verlaufe der Jahrzehnte verändert oder haben zumindest neue Facetten angenommen. In ihnen widerspiegeln sich sowohl die allgemeinen gesellschaftlichen Veränderungen und Tendenzen als auch die Entwicklungen der Medizin. Holzschnittartig begegnen wir im Alltag den folgenden stereotypenartigen Rollenbildern:

\section{Patient als Fürsorgeempfänger}

Als eines der ursprünglichsten Rollenbilder sahen sich Patientinnen in der Vergangenheit im wesentlichen als «bedürftige Empfänger» der ärztlichen und pflegerischen Fürsorge. Der Patient kam mit einer passiven Opferhaltung ins Spital. Beim Eintritt gab er nicht nur den Koffer, den er bis dorthin selbst getragen hatte, in die Hand der ihn empfangenden Krankenschwester $a b$, sondern gleich auch noch seine Selbstverantwortung: «Hier bin ich, macht mich gesund!» Die Arbeit der Pflegenden quittierte er im positiven Fall mit den Worten: «Es waren liebe Schwestern.» Wenn er schon gegenüber den Krankenschwestern die Haltung eines unmündigen Kindes einnahm, ist verständlich, dass er den Arzt als «Halbgott in Weiss» verehrte.

\section{Patient als interessanter medizinischer Fall}

Mit den rasanten Fortschritten der Medizin, die eine Spezialisierung und Arbeitsteilung im Spitalbetrieb mit sich brachten, verlagerte sich der Blick von der Bedürftigkeit der Patienten auf faszinierende Diagnose- und Therapiemöglichkeiten. Die Patientin wurde zum «medizinischen Fall». Im Spital sprachen nicht nur Ärzte, sondern auch Pflegende von der «Hüfte in Zimmer 26». Arztstellen im Universitätsspital waren und sind begehrt, weil es dort ein «interessantes Patientengut» hat. Patienten waren stolz, wenn sie vom Professor den Studierenden als «interessanter Fall» vorgestellt wurden. Der Patient wurde in dieser Optik mehr Objekt (Träger einer Krankheit) als Subjekt (kranke Person), sowohl aus der Sicht der Ärzte als auch in seinem Selbstbild. Die Krankheit wurde unabhängig von der erkrankten Person als ein isolierter, begrenzter Prozess identifiziert und gezielt (und häufig auch sehr erfolgreich) behandelt.

\section{Patient als konsumierender Kunde}

Vielleicht als Reaktion auf dieses Bild tauchte in neuerer Zeit der Begriff «Kunde» in den Spitälern auf. Nach diesem Verständnis ist die Patientin primär das, was sie ausserhalb des Gesundheitswesens schon immer war, nämlich eine Kundin, und fordert als solche auch im Gesundheitswesen das, was ihr andere Dienstleistungsbetriebe selbstverständlich bieten. Für Wartezeiten (nirgends gibt es so viele Wartezimmer wie im Gesundheitswesen), unverständliche oder gar unfreundliche Antworten oder für mässig warmes Essen hat der Kunde wenig Verständnis. Gesundheit erhielt den Charakter eines Konsumguts. Der Patient wurde zum konsumierenden «Kunden». Spitäler unternahmen Anstrengungen, die Kundenorientierung ihrer Mitarbeitenden zu verbessern. 


\section{Das Bild vom Patienten als Kunden geht nicht auf}

Beim näheren Hinschauen zeigt sich, dass dem Patienten drei für den Kunden wesentliche Merkmale fehlen:

- Der Kunde wählt den Anbieter selbst aus, wogegen es beim Patienten in der Regel der Hausarzt ist, welcher ihm die Spitalwahl abnimmt. Bei einer Einweisung per Rettungssanität bestimmt höchst selten der Patient das Spital.

- Die Kundin entscheidet, z.B. aufgrund eines Kostenvoranschlags, selbst, welche Leistungen sie kaufen will, wogegen im Spital die Ärzte und Pflegenden bestimmen, was die Patientin nötig hat.

- Der Kunde trifft seine Entscheidungen aufgrund des Preis-Leistungs-Verhältnisses, wogegen für den Patienten nach dem Eintritt ins Spital der Preis nie eine Rolle spielt, da ja die Krankenversicherung die Rechnung bezahlen wird. Noch schwieriger ist es bei der Evaluation der Qualität medizinischer Leistungen. Sie entzieht sich dem Patienten in der Regel und basiert meist auf Vertrauen.

Die Patientin kann diese drei wesentlichen Elemente der Kundenautonomie nicht wahrnehmen. Sie fühlt sich diesbezüglich eher abhängig. Der Objektstatus wird so nicht überwunden. Eine zunehmende Zahl von Patienten versucht deshalb, ihren Autonomieanspruch auf eine Art und Weise zur Geltung zu bringen, die der spezifischen Situation eines Patienten besser gerecht wird:

a Der Fragebogen des PickerInstituts wurde aus der Sicht der Patienten, d.h. von Patienten entwickelt. Er wird in den USA und verschiedenen europäischen Ländern verwendet.

b Bei diesen Fragen geht es um Leistungen und Informationen, die der Patient als Kunde auch ausserhalb des Gesundheitswesens von einer Dienstleistungsfirma erwartet. Hier ist der Patient konsumierender Kunde («konsumbezogene Fragen»).

c Bei diesen Fragen geht es um Leistungen und Informationen, die der Patient (oder seine Angehörigen) für ein aktives Mitwirken am Gesundungsprozess oder für eine aktive Auseinandersetzung mit der Krankheit benötigen. Es handelt sich um patientenspezifische Ansprüche, die für gesunde Kunden ausserhalb des Spitals inhaltlich nicht sehr relevant sind («partizipationsbezogene Fragen»).

In diesem Zusammenhang sind die Resultate der jährlich in allen öffentlichen Spitälern der Kantone Bern, Solothurn und Zürich durchgeführten «Picker»-Patientenbefragunga interessant. Alles in allem sind die Patienten im Kanton Zürich zu 79\% mit den Spitälern zufrieden. Wenn nur die konsumorientierten Themen ${ }^{\mathrm{b}}$ beachtet werden, liegt die Zufriedenheit sogar bei $89 \%$. Wenn aber nur die Antworten auf partizipationsorientierte Fragen ${ }^{c}$ ausgewertet werden, beträgt die Zufriedenheit nur noch 70\%. Auf die konkrete Frage «Konnten Sie bei Ihrer Behandlung genügend mitbestimmen?» antworteten nur 63\% der Patienten positiv. Der durchschnittliche Anteil der in dieser Hinsicht unzufriedenen Patienten im Kanton Zürich liegt bei $37 \%$ !
Ein Drittel der heutigen Patienten hat Mühe mit der Vorstellung, mehr oder weniger fremdbestimmt untersucht, behandelt und gepflegt $\mathrm{zu}$ werden. Und genau das ist das gemeinsame Merkmal der Patientenbilder «Fürsorgeempfänger», «Medizinischer Fall» und «konsumierender Kunde». Die mit den gebotenen Partizipationsmöglichkeiten unzufriedenen Patienten entsprechen einem anderen Bild. Sie sehen sich als «verantwortlich Mitwirkende». Sie sind sich bewusst, dass Gesundheit nicht einfach fertig fabriziert konsumiert werden kann, sondern in einem Prozess entsteht, der sich in ihrem eigenen Körper abspielt, an dem sie aktiv beteiligt sind, der unmittelbar etwas mit ihnen selbst zu tun hat. Autonomie erfordert aus ihrer Sicht Informationen, die ihnen ermöglichen mitzubestimmen, was mit ihnen und für sie getan werden soll. Autonomie bedeutet für sie, soviel wie möglich aktiv am Gesundungsprozess beteiligt zu sein. Für ihr Mitwirken übernehmen sie auch Verantwortung. Das unterscheidet sie wesentlich von den anderen drei Patientenbildern, entspricht aber deutlicher dem ausserhalb des Gesundheitswesens heute in unserem Kulturkreis allgemein relevanten Menschenbild.

\section{Aktuelle Vielfalt von Selbstbildern der Patienten}

Die relativen Häufigkeiten der vier beschriebenen Patientenbilder haben sich im Verlauf der letzten Jahrzehnte verändert. Während noch in der Mitte des letzten Jahrhunderts der Typus des passiven Fürsorgeempfängers im Vordergrund stand, scheint heute keines dieser Bilder zu dominieren. Wir haben es vielmehr mit einer Vielfalt von nebeneinander bestehenden Patientenbildern zu tun (Abb. 1).

\section{Patientenbild als Ausdruck des medizinischen Fortschritts}

Das neue Bild vom Patienten als einem verantwortlich Mitwirkenden spiegelt nicht nur die allgemein an Bedeutung zunehmende Autonomieforderung wider, sondern kann auch als Reaktion auf die Entwicklung der modernen Medizin verstanden werden: Solange der Arzt einen grossen Teil des gesamten Wissens beherrschte, das für die erfolgreiche Behandlung einer Patientin zur Verfügung stand, lag es auch für die Patientin auf der Hand, den Arzt als quasi allmächtig und sich selbst als von dessen Fürsorge vollkommen abhängig zu sehen. Mit 


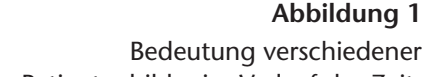
Patientenbilder im Verlauf der Zeit.

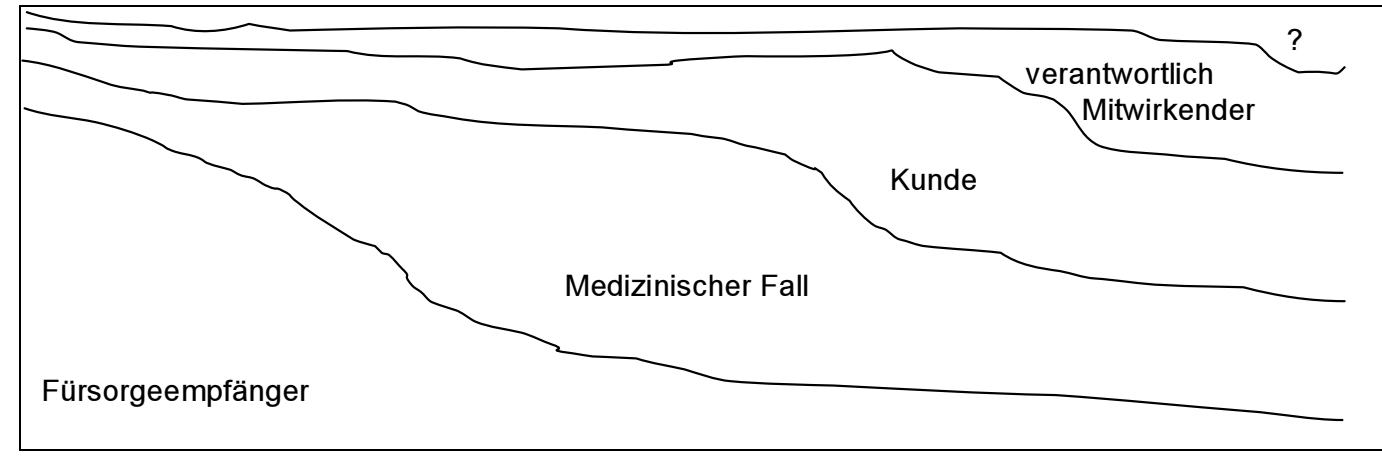

1950

1990

2000 der zunehmenden Spezialisierung der Medizin beherrschte der einzelne Arzt einen immer kleineren Teil des insgesamt zur Verfügung stehenden Wissens. Interdisziplinäre Prozesse der Patientenuntersuchung und -behandlung wurden die Regel. Die elektronische Revolution der vergangenen Jahre mit Internet und Onlinedatenbanken macht zudem medizinisches Wissen für jedermann greifbar. Patienten mit sehr spezifischen Krankheitsbildern, die sich oftmals in Internetselbsthilfegruppen zusammenfinden, sind heutzutage schon gelegentlich über neueste Studienergebnisse besser informiert als ihr Arzt. Der Umgang mit Information und Desinformation, mit Gewichtung einzelner Erkenntnisse und ihrer dem Einzelfall angemessenen Synthese ist eine neue zentrale Herausforderung für die Medizin. Während man früher froh war, überhaupt eine wirksame Therapie zu kennen, steht die Ärztin heute zunehmend vor Alternativen und damit vor der Entscheidung, welcher Weg für den konkreten Patienten der beste sei - der beste im Sinne der Koordination der Untersuchungen und Behandlungen sowie im Sinne der Sicherstellung von Evidence-based Medicine oder im Sinne der Zweckmässigkeit, Wirksamkeit und Wirtschaftlichkeit der Leistungen. Und genau hier wollen ein Drittel der Patientinnen als verantwortlich Mitwirkende mehr mitbestimmen, als man ihnen das in der heutigen Spitalpraxis zugesteht. Johann Steurer sagt treffend: «Der Arzt ist der Experte für das Wissen; der Patient ist Experte für seine Präferenzen» [1]. Wir sind gewohnt, zwar unter Berücksichtigung des Wissens, aber vor allem aufgrund unserer eigenen Präferenzen zu entscheiden!

\section{Arzt-Patienten-Beziehung}

Für die Gestaltung der Arzt-Patienten-Beziehung ergeben sich aufgrund der verschiedenen Patientenselbstbilder entsprechend unterschiedliche
Ausgangslagen. In jedem Fall wäre im Rahmen der Anamnese zunächst das Selbstbild des Patienten zu erfragen und damit auch die Erwartungen des Patienten an die Rolle des Arztes.

Mit den Patientenselbstbildern des Fürsorgeempfängers und des medizinischen Falls scheint man in den Spitälern wenig Mühe zu haben. Auf die Erwartungen des Patienten als Kunden haben sich verschiedene Spitäler mit Kundenorientierungsschulungen des Personals ausgerichtet. All das geht aber zuwenig auf die Erwartungen des Patienten als verantwortlich Mitwirkender ein: Wenn die Patienten den Eindruck haben, sie würden als Subjekte zuwenig ernst genommen und miteinbezogen, bringt es nicht viel, wenn sie als Objekte noch ein bisschen besser bedient werden. Im Gegenteil: Die unbefriedigende Erfahrung des Patienten, seine Subjektautonomie nur bei vergleichsweise nebensächlichen Aspekten wie der Wahl des Essens ernst genommen zu sehen, bei der Wiederherstellung seiner Gesundheit selbst jedoch nicht mitbestimmen und autonom mitwirken zu können, kann alle anderen Verbesserungsbemühungen des Spitals überschatten.

\section{Autonomie und Abhängigkeit}

Der Patient als verantwortlich Mitwirkender will - im Rahmen seiner situativen Möglichkeiten - aktiv im Mittelpunkt des Prozesses stehen, der zu seiner Gesundung führt oder in dessen Verlauf er einen guten Umgang mit seiner Krankheit entwickelt:

- Im ausgesprochen arbeitsteiligen, interdisziplinären Prozess eines Spitalaufenthalts erwartet der Patient als Mitwirkender ein transparentes Prozessdesign, das er nachvollziehen und verstehen kann.

- Er zweifelt an der Professionalität des Spitals, wenn er auf die gleiche Frage an zwei Mitarbeitende unvereinbare oder offensichtlich 
nicht aufeinander abgestimmte Antworten erhält.

- Es stört ihn, wenn etwas mit ihm gemacht wird, dem er nicht nach zuvor erfolgter Information zugestimmt hat.

- Er erwartet begründete Vorschläge, wenn möglich Alternativen mit Vor- und Nachteilen, Chancen und Risiken.

- Wenn er nicht selbst zu entscheiden wagt, ist er dankbar für professionelle Hilfe bei der Entscheidungsfindung. Hierin liegt auch ein Potential für eine moderne Patientenschulung.

Die Patientin als verantwortlich Mitwirkende will trotz verschiedenen Abhängigkeiten ihre Autonomie bewahren, ernst genommen und miteinbezogen werden. Das relative Gewicht dieses Patientenbildes kann sich je nach Krankheit, Alter oder auch im Verlaufe eines Spitalaufenthalts ändern. Aber auch eine schwerkranke Patientin nimmt ihre Autonomie durchaus wahr, wenn sie aufgrund ihrer Situation die Ärztin bittet, in ihrem (der Patientin) Sinn zu entscheiden. Die autonome Delegation der Autonomie ist auch eine Form von Autonomie.

Ein Blick über das Gesundheitswesen hinaus - und von dort kommen ja all unsere Patientinnen - lässt vermuten, dass der Anteil dieses Patientenselbstbildes in den nächsten Jahren generell zunehmen wird. Ein Grossteil der Patienten will besser verstehen, was im Spital abläuft, und will mitentscheiden, was im Spital mit ihnen geschieht. Selbstverständlich bleibt der Patient in einer gewissen Abhängigkeit vom Spital, wie das beim Fürsorgeempfänger und beim medizinischen Fall das Hauptcharakteristikum war, aber was der Soziologe Richard Sennett [2] über obdachlose Jugendliche sagt, gilt auch für die Patienten: Sie wehren sich heute dagegen, «Zuschauer ihrer eigenen Bedürftigkeit zu werden». Spitäler dürfen nicht den Fehler machen, einerseits $\mathrm{zu}$ versuchen, ihre Klienten als ganze Menschen zu behandeln, andererseits aber nicht zu akzeptieren, dass diese als ganze Menschen eben in der Lage sind, über die Bedingungen ihrer Abhängigkeit selbst mitzubestimmen. Die zentrale Frage lautet daher: Wie können Patienten Unterstützung erfahren und zugleich in ihrer Autonomie ernst genommen werden?

Es gilt, im Spital die Sensibilität für die Spannung zwischen Autonomie und Abhängigkeit der Patienten zu fördern. Dabei geht es um nichts weniger als die Grundhaltung der Pflegenden und der Ärzte den Patienten gegenüber. Wenn erfolgreiche Ärzte berichten, sie hätten mit einer paternalistischen Haltung gute

Tabelle 1

\begin{tabular}{|c|c|}
\hline Selbstbild des Patienten & Rolle des Arztes aus der Sicht des Patienten \\
\hline Fürsorgeempfänger & Fürsorgeempfänger \\
\hline bedürftig & weiss, was ich brauche \\
\hline hilflos & macht es schon richtig \\
\hline dankbar & kann nichts dafür, wenn es trotz allem nicht besser wird \\
\hline medizinischer Fall & medizinischer Fall \\
\hline «nur» Träger der Krankheit, nicht krank gewordener Mensch & weiss alles über meine Krankheit \\
\hline \multirow[t]{2}{*}{ Gesundheit ist eine Frage des wissenschaftlichen Fortschritts } & kennt die neusten Therapien aus den USA \\
\hline & hat Beziehungen zur Universität, zu Spezialisten \\
\hline Kunde & Kunde \\
\hline ärztliche Leistungen als Konsumgüter & lässt mich nie warten \\
\hline Gesundheit ist nur eine Frage des Geldes & ist freundlich \\
\hline \multirow[t]{3}{*}{ hasst Wartezeiten und jede Art nicht zuvorkommender Behandlung } & interessiert sich für mich \\
\hline & bedient mich in schönen Räumen \\
\hline & behandelt mich nach dem Motto: Ich als Kunde bin König \\
\hline verantwortlich Mitwirkender & verantwortlich Mitwirkender \\
\hline will informiert werden & nimmt mich ernst \\
\hline will mitentscheiden & sagt, dass er auch nicht alles weiss \\
\hline \multirow[t]{3}{*}{ krank sein und gesund werden als persönlicher Prozess } & fragt mich um meine Meinung \\
\hline & $\begin{array}{l}\text { informiert mich umfassend und verständlich über Vor- und Nachteile, } \\
\text { Chancen und Risiken }\end{array}$ \\
\hline & lässt mich ohne Erwartungsdruck selbst entscheiden \\
\hline
\end{tabular}


Erfahrungen gemacht und damit sogar den Erwartungen der Patienten entsprochen, so ist diese Wahrnehmung verständlich und entspricht als adäquate Antwort den vor allem früher dominierenden Patientenbildern des «Fürsorgeempfängers» und des «medizinischen Falls». Aber schon beim Bild des Patienten als «Kunden» kommt eine paternalistische Haltung ins Wanken. Beim Patienten als «verantwortlich Mitwirkendem» ist sie fehl am Platz. Der mentale Schritt vom Spitalpaternalismus weg hin zur Patientenautonomie wird zwingend. Die Herausforderung besteht in der Wahrung der Menschenwürde des Patienten im Spannungsfeld zwischen seinem Autonomieanspruch und seiner Abhängigkeit. Dies ist das Grundanliegen moderner Medizinethik.

\section{Normatives Potential des Autonomiemodells}

Wenn wir davon ausgehen, dass zu einem gesunden Leben auch gehört, Selbstverantwortung wahrzunehmen, und dass Gesundheit oder eine gesunde Haltung zur Krankheit etwas mit der reflektierten Haltung im Spannungsfeld zwischen Fremd- und Selbstbestimmung zu tun haben, kommt dem Patientenbild des verantwortlich Mitwirkenden normative Bedeutung zu. Im Unterschied zu den anderen drei Selbstbildern erfüllt es den Autonomieanspruch, der sich direkt vom Grundpostulat der Menschenwürde ableitet. Die anderen drei Bilder haben aus der Sicht des modernen Verständnisses von Medizinethik tendenziell einen pathologischen Beigeschmack. Selbst wenn die individuelle
Autonomiefähigkeit einer Patientin weit dem grundsätzlichen, für alle Menschen geltenden Autonomieanspruch hinterherhinkt, kann es für die Ärztin immer nur darum gehen, den mutmasslichen Willen dieser Patientin zu respektieren. Die Richtlinien der Schweizerischen Akademie für Medizinische Wissenschaften sind hier absolut eindeutig.

Obwohl es schon kundenfreundlich ist, wenn ein Arzt nach den Rollenerwartungen eines Patienten fragt und versucht, diese Erwartungen zu erfüllen, mag die flexible, situative Rollenübernahme nicht befriedigen. Vorzuziehen ist ein ärztliches Verhalten, das einem Patienten mit dem Selbstbild des hilflosen Fürsorgeempfängers, des medizinischen Falls oder des konsumierenden Kunden hilft, sein Autonomiepotential gerade auch als Patient zu erkennen und zu nutzen, um ihn als verantwortlich Mitwirkenden zu gewinnen. Unabhängig vom Selbstbild der Patientin sollte die Förderung ihrer Autonomiefähigkeit zum Selbstverständnis der Ärzte und aller anderen Mitarbeitenden im Spital gehören.

Ich danke Dr. Heinz Rüegger und Dr. Ludwig T. Heuss für die konstruktiv-kritische Durchsicht des Manuskripts und für ihre Anregungen.

\section{Literatur}

1 Büchi M, Bachmann LM, Fischer JE, Peltenburg M, Steuerer J. Alle Macht den Patienten? Vom ärztlichen Paternalismus zum Shared Decision Making. Schweiz Ärztezeitung 2000;81(49):2776-80.

2 Sennett R. Respekt im Zeitalter der Ungleichheit. Berlin: Berlin Verlag; 2002. 